\title{
Anthós
}

2014

\section{A Comparison of Different Treatments for Mesothelioma}

Samira Rezaei

Portland State University

Follow this and additional works at: https://pdxscholar.library.pdx.edu/anthos

Part of the Alternative and Complementary Medicine Commons, and the Cancer Biology Commons Let us know how access to this document benefits you.

\section{Recommended Citation}

Rezaei, Samira (2014) "A Comparison of Different Treatments for Mesothelioma," Anthós: Vol. 6: Iss. 1, Article 15.

https://doi.org/10.15760/anthos.2014.137

This open access Article is distributed under the terms of the Creative Commons Attribution-NonCommercialShareAlike 4.0 International License (CC BY-NC-SA 4.0). All documents in PDXScholar should meet accessibility standards. If we can make this document more accessible to you, contact our team. 


\section{A Comparison of Different Treatments for Mesothelioma}

Samira Rezaei

The goal of this research was to survey the literature of studies on mesothelioma treatments and compare their rates of success. The focus was on radiation therapy which is used with chemotherapy or surgery treatment for this specific type of cancer. The research is based on the chosen treatments of thirteen patients who shared their stories on different websites (Table 1). The number of survived years for these patients was compared. The results show that the average number of years survived in patients who used mind-body therapy is greater than other patients who use radiation therapy as palliation with chemotherapy, surgery treatment or immune therapy as treatment of their disease. The significance of this research was to find the best treatment for mesothelioma cancer and find the advantage of using radiation therapy as treatment in this specific type of cancer. This was done by comparing the rate of patients who survived longer with the survey in 1990 (Ball \& Cruickshank, 1990).

\section{Introduction}

The definition of cancer is a disease in which abnormally developed cells start to divide out of control. Cancer usually is named after the type of cell or the organ in which they begin. There is a line of cells contained in the abdomen (peritoneum), chest (pleura) and in space around heart called the mesothelium. These cells protect organs by secreting a special fluid that allows the organs to move. For example, this fluid makes it easier for heart to pump outward. Malignant mesothelioma has been categorized based on the position 
of the migrant cells: pleural mesotheliomas, which start in the chest, peritoneal mesotheliomas which start in the abdomen, and pericardial mesotheliomas which start in the outer layer around heart. Additionally, mesothelioma is named based on the shape of the cancer when viewed under a microscope. An epithelioid sarcoma is a rare mesenchymal soft tissue tumor with an epithelioid pattern (FanburgSmith, Fetsch, Miettinen, Shmookler \& Virolainen, 1999). And sarcomatoid carcinoma is a relatively uncommon form of cancer whose malignant cells have histological, cytological, or molecular properties of both epithelial tumors (carcinoma) and mesenchymal tumors.

Thousands of workers and servicemen have become disabled or died because of the health effects of asbestos. The expectation is that many more will be affected in near future (Craighead \& Gibbs, 2008). Asbestos fibers are a risk factor of mesothelioma, as they can travel to the ends of the small airways and reach the cell lining of the lungs during normal breathing. Asbestos fibers are naturally occurring minerals. They are resistant to heat and corrosion. They are used in products such as insulation for pipes (steam line), floor tiles, building materials, and in vehicle brakes and clutches (Craighead \& Gibbs, 2008).

What is yet to be understood is why mesothelioma is found in children as well. Although the average age of diagnosis of mesothelioma is sixty-five years, and is usually found in men, it has been found in children as well. The risk factor for mesothelioma in children has not been identified definitively. Children may also experience direct exposure to asbestos in the home. This may occur where there are damaged or worn asbestos fibers that have been released into the air from old insulation or other old building materials. In this situation, the child might inhale the fibers, which would then become lodged in the chest or abdominal area, eventually leading to the formation of mesothelioma cancer. Children can also suffer secondary exposure. This can occur when someone else in their 
household works with asbestos - which is rare these days - and brings home toxic dust on their clothes or in their hair. The child can breathe in these fibers and may eventually get sick because of the exposure to the hazardous substance (American Cancer Society, 2013).

\section{Diagnosis}

Normally, the cancer is diagnosed by an expert who looks at samples of cancer, which could be cell or tissue, under microscope. In some cases, lab tests of the cell proteins, DNA, and RNA can be used for diagnosis as well. There are three ways to diagnose mesothelioma cancer.

\section{Screening such as X-ray, Fluroroscopy, CT}

The standard way of diagnosis is through imaging. X-rays for diagnostic procedures or research are produced by accelerating electrons using a high voltage and allowing them to collide with a metal target (Maniwa, Nishimura, Ohno, Takata \& Yoshimura, 2008). $\mathrm{X}$-rays are produced when the electrons suddenly decelerate upon collision with the metal target. These X-rays are products of what is commonly called braking radiation. If the bombarding electrons have sufficient energy, they can knock an electron out of an inner shell of the target metal atoms. When a metal target is bombarded by highenergy electrons, X-rays are emitted. An X-ray spectrum typically consists of a broad continuous spectrum and a series of sharp lines. Where lines are depends on the metal is used. These X-rays are called characteristic X-rays.

There are different imaging modalities: radiography, fluoroscopy, computed tomography, nuclear medicine, magnetic resonance imaging, and ultrasound.

In radiography, standard X-ray is used. The radiation will ionize and it is the initial screening study of bone, chest, and abdomen for mammography. In radiography, air (black), fat (dark gray), 
Anthós, Vol. VI, Issue 1

water/tissue (gray), bone (light gray), metal (white) appear in different colors.

In fluoroscopy, the real-time X-ray uses ionizing radiation. It is utilized for X-ray guided procedures, such as GL studies, angio/interventional. Radiography shows the same tissues in the same color.

In computed axial tomography, ionizing radiation is used and the computer reconstructs cross-sectional (axial) images. It is the initial screening for brain pathology and also for the chest, abdomen and pelvis.

\section{MESOMARK}

The MESOMARK assay is one of the most promising diagnostic markers for mesothelioma cancer (Alexander, 2006; Hino \& Maeda, 2006 \& Hino \& Shiomi, 2007).

In a blood test called the MESOMARK assay, the levels of soluble mesthelin-related peptide (SMRP) is measured. SMRP is a protein released into the blood by cancerous mesothelioma cells.

Cancer progresses through a staging system which corresponds to the severity of the cancer. This is based on the size and extent of the primary tumor and whether or not cancer has spread in the body. These stages help the medical doctors to plan appropriate treatment. It can be used to deduce a patient's cancer prognosis. It could also help provide a platform for the exchange and comparison of information between patients (Ebara et al, 2012). There are different ways to determine the stage. Physical exams, imaging studies, laboratory tests, pathology and surgical reports are the ways to track the stages of cancer. The most widely used cancer staging system is TNM. It is based on the size and reach of the primary tumor $(\mathrm{T})$, the amount of spread to lymph node $(\mathrm{N})$, and the existence of metastasis (M). Five stages are explained in the TNM systems. Usually mesothelioma is diagnosed in stage three and four with symptoms. Pleural mesothelioma symptoms are characterized by pain in the lower back 
or at the side of the chest, shortness of breath, cough, fever, sweating, tiredness, weight loss, hoarseness, swelling of the face and arms.

\section{Treatment}

There are different treatments for surviving patients with mesothelioma. These include the most used ones, which are radiation therapy, surgery, targeted therapy, immune therapy, and chemotherapy. In the mesothelioma, radiation therapy is used as a less painful alternative. Radioactive particles are used in different ways for treatment of this cancer. These are: radiation therapy, chemotherapy, and immunotherapy (for high dose chemotherapy).

\section{Surgery}

Surgery is another option for a person who diagnosed with mesothelioma. There are different types of surgery. Curative surgeries are used in the early stage of mesothelioma, such as pneumonectomy, extra pleural and pleurectomy. Palliative surgeries are another type of surgery which is used to relieve mesothelioma symptoms like pain and other problems associated with mesothelioma disease, such as pleurocentesis, paracentesis, and pleurodesis.

\section{Targeted Therapy or Molecularly Targeted Therapy}

Molecularly targeted therapy is another option of treatment that uses drugs or other substances to identify and attack specific cancer cells (National Cancer Institute). This type of therapy has fewer side effects than other types.

\section{Chemotherapy}

Chemotherapy is a type of treatment in which the cancer cells are killed. This type of treatment could be useful depending on the stage of cancer and where a patient is in treatment process. It could be used for curing the cancer, preventing it from spreading, slowing its growth, killing cancer cells that may have spread to other parts of 
body, and relieve the symptoms caused by cancer (American Cancer Society.). Many clinical trials have tested the numerous chemotherapeutic agents. The response rate doesn't exceed more than $20 \%$ for most of the investigated regimens (Maniwa, Nishimura, Ohno, Takata, \& Yoshimura, 2008).

\section{Immune Therapy}

Immune therapy is a type of injection chemotherapy which is used for high-dose chemotherapy. In this type of treatment, the body's immune system is used to aid the fight against the cancer. Common types of immunotherapy include monoclonal antibodies, cancer vaccines, and non-specific immunotherapies. In immunotherapy, radioactive materials are placed directly into the chest or the abdomen at the site of the cancer. The radiation will move only a very short distance, which limits the possible damage to nearby healthy tissues. This is the method that is normally used for mesothelioma.

\section{Radiation Therapy}

Radiation therapy is a treatment in which beams of high-energy particles are directed at cancer cells to reduce the potency of cancerous cells. There are different types of radiation therapy, but two are specifically used in treating mesothelioma. One is called external beam radiation therapy (EBRT). In this regimen, the beam source is usually on the outside of the patient's body. A machine is used to produce this type of beam. Another type of radiation therapy is brachytherapy, in which the radiation source is placed close to the surface of the body or within a body cavity.

In External Beam Radiation Therapy (EBRT), the source of radiation may be outside the body of the patient (external radiation therapy) or it may be an isotope that has been implanted into abnormal tissue or a body cavity. Modern radiation therapy primarily uses high-energy $\mathrm{X}$-rays or gamma rays with peak photon energies, exceeding 1 mega electron volte. Brachytherapy's radiation sources 
are either low energy photon sources (for LDR, or low dose rate applications) or high energy photon sources (used for high dose rate brachytherapy). Beta brachytherapy is used for intravascular brachytherapy for prevention of restenosis and ophthalmic applicators).

Radiation therapy usually is used in two different ways. First, it is used to cure cancer. In this way the goal is to destroy tumors that have not spread to other body parts and reducing the risk that cancer will return after surgery or chemotherapy. Second, radiation therapy is used to reduce symptoms. In this way, shrinking tumors will improve quality of life. For example, in mesothelioma, the symptoms cause shortness of breath. The pain can be alleviated, however, by reducing the size of tumors. Radiation is a type of energy in motion. It can be in form of fast moving particles of energy waves. There are many types of radiation in everyday life such as TV, infrared, visible light, UV, X-Rays.

Atoms are not stable. The excess energy contained in an unstable atom is released in the form of a few basic particles and energetic waves. Alpha particles are the heaviest particles and are produced when the heaviest elements decay. Beta particles are much lighter than alpha particles. Another high energy particle is the gamma ray. It is an energetic photon, or light wave, in the same electromagnetic family as light and X-rays, but is much more energetic and harmful. Gamma rays are also capable of damaging living cells, as they slow down by transferring energy to surrounding cell components.

The major difference between gamma rays and X-rays is that gamma rays have more energy. For example, gamma rays have over a trillion times more energy than typical radio waves. When gamma rays and X-rays interact with the human body, ionization happens, because those waves have sufficient energy to dislodge electrons. The radiation imparts enough energy to an orbital electron in an atom for the electron to free itself. One of the problems with ionization is that freed electrons can cause damage to living cells or DNA. If the 
Anthós, Vol. VI, Issue 1

radiation dose is small, the body can repair itself but a high dose can cause biological damage.

The way ionizing radiation is used to cure cancer is that it minimizes the dose to the healthy tissue, while maximizing the dose to cancerous tissue. These doses are delivered in small fractions. Such doses take advantage of the fact that healthy tissues have better repair mechanisms for radiation damage than cancer cells have. A normal, self-repairing cell cycle consists of four phases: mitosis, which takes one hour, synthesis, which takes six to fifteen hours (depending on what kind of cell it is), G2, which takes 3 to 4 hours, and G1, which is variable.

Treatment planning systems contain five steps which are positioning, contouring, beam arrangement, optimization and dose calculation and display.

The problem with radiation therapy for mesothelioma is that the cancer is in close proximity to the heart and lungs and it is risky to provide the kind of high-dose, intensive therapy needed to shrink the tumor. IMRT (Intensity-Modulated Radiation Therapy) can more accurately target cancer cells and avoid healthy tissue. It may provide better results when performed by experienced clinicians. 
Table 1. Number of Years Patients Survived when Using Different Treatments for Mesothelioma.

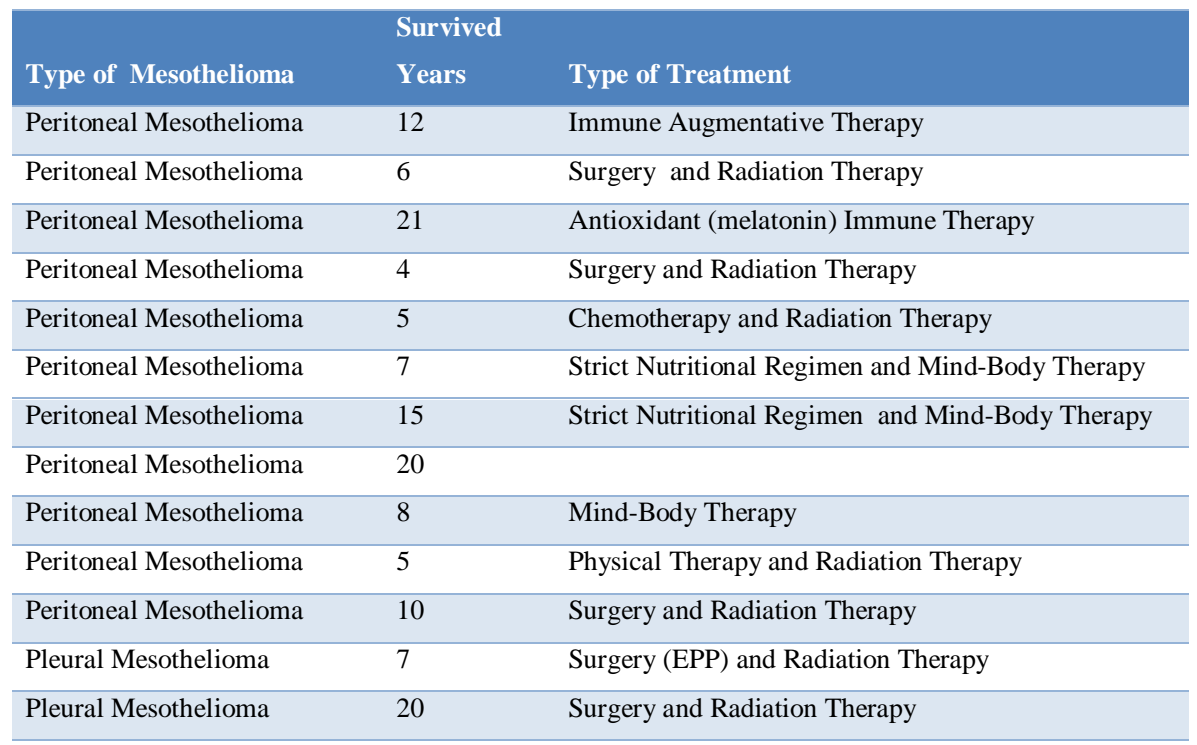

Table 2. Stem Plot - Treatments vs. Years Survived.

\begin{tabular}{|c|c|c|}
\hline Immune Therapy & Surgery & $\begin{array}{c}\text { Mind-Body } \\
\text { Therapy }\end{array}$ \\
\hline 12 & 6 & 7 \\
\hline 21 & 4 & 15 \\
\hline 5 & 10 & 8 \\
\hline & 7 & \\
\hline & 20 & \\
\hline
\end{tabular}

In the research presented above, three different treatments were compared (Table 2). These were: immune therapy, surgery, and mindbody therapy. The averages of the years the patient survived past the time the cancer is diagnosed was measured. Averages are shown in the following graph. 
Anthós, Vol. VI, Issue 1

Figure 1. Different Treatments vs. Years Survived.

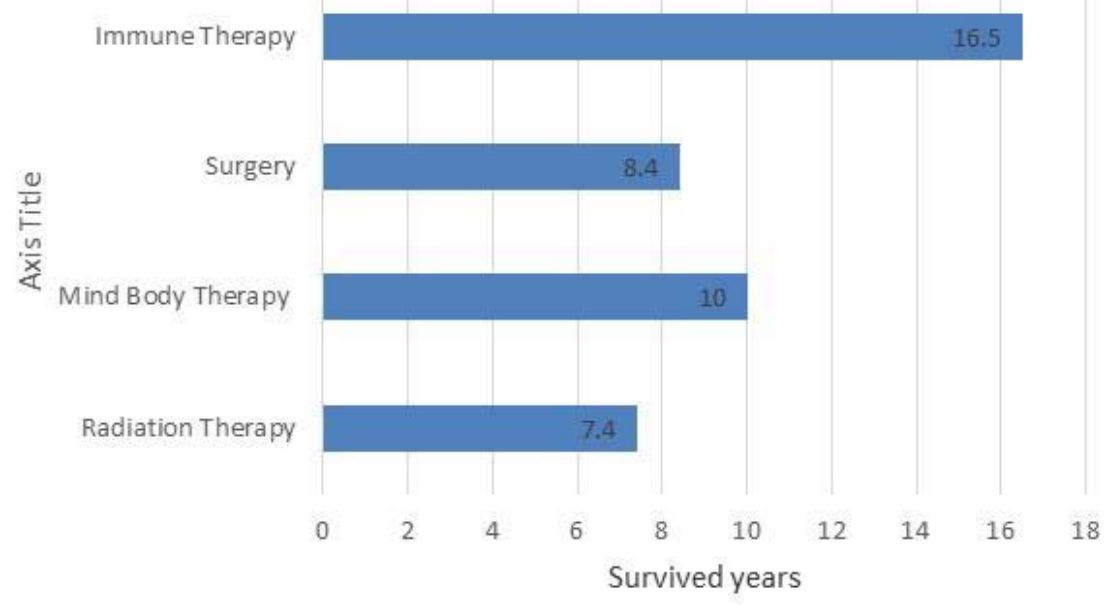

Figure 1 shows that the average number of years survived in patients who used immune therapy is greater than the other patients who used surgery and radiation therapy.

\section{Conclusion}

One of the rarest forms of cancer is mesothelioma. The cancer cells are found in pleura or peritoneum (Carbone, Pass \& Vogelzang, 2005). The fact about Mesothelioma is that by the time the symptoms appear the disease is often advanced. Regardless of the type of treatment, in an advanced situation it is difficult to treat it. According to a survey in 1990, the median amount of time the patient survived since the time of diagnosis, out of 38 patients, was 9 months, with an estimated 2-year survival rate of $16 \%$. Radiation therapy is more useful for palliation (Ball \& Cruickshank, 1990). This research introduced mesothelioma cancer and then further explained the different methods of diagnosis. The imaging methods in which radiation particles are used were delineated. The different treatments for mesothelioma were introduced. Patients who were treated with 
immune therapy, surgery, and mind body therapy were compared in terms of the number of years survived. Also, the advantages and practical aspects of radiation therapy were explained. In summary, this research shows that patients who suffered from mesothelioma cancer and chose the immune therapy treatment to treat it have survived longer in comparison to patients who used other methods of treatment.

\section{References}

Alexander, R. H., Bera, T. K., Ho, M., Hassan, R., Nagata, S., Onda, M. \& Pastan, I. (2006). Megakaryocyte Potentiation Factor Cleaved from Mesothelin Precursor is a Useful Tumor Marker in the Serum of Patients with Mesothelioma. Clinical Cancer Research, 12, 4225-4231.

American Cancer Society. (2013). Learning about Chemotherapy Treatment and Radiation Therapy for Malignant Mesothelioma. Retrieved from http://www.cancer.org/treatment/treatmentsandsideeffects/treatmenttypes/ chemotherapy/understandingchemotherapyaguideforpatientsandfamilies/ understanding-chemotherapy-learning-what-is-it-how-it-works

American College of Radiology. Radiation Safety. Retrieved from http://www.acr. org/quality-safety/radiology-safety/radiation-safety

Asbestos HUB. (2010). Malignant Mesothelioma: Overview Guide. Retrieved from http://www.asbestoshub.com/2010/07/01/overview-guide/

Ball, D. L. \& Cruickshank, D. G. (1990). The Treatment of Malignant Mesothelioma of the Pleura: Review of a 5-Year Experience, with Special Reference to Radiotherapy. American Journal of Clinical Oncology, 1, 4-9. Cancer.net. Side Effects of Radiation Therapy. Retrieved from http://www.cancer .net/navigating-cancer-care/how-cancer-treated/radiation-therapy/side-effectsradiation-therapy

Carbone, M., Pass, H. \& Vogelzang, N. (2005). Malignant Mesothelioma: Advances in Pathogenesis, Diagnosis, and Translational Therapies. New York: Springer.

Craighead, J. E., \& Gibbs, A. R. (2008). Asbestos and Its Diseases. New York: Oxford University Press.

Ebara, T., Ishikawa, H., Maeno, T., Nakano, T Okonogi, N., Ueno, M., Yoshida, D., (2012). A Seven-Year Disease-Free Survivor of Malignant Pleural Mesothelioma Treated with Hyperthermia and Chemotherapy: A Case Report. Journal of Medical Case Reports, 6(427). Retrieved from http://www.jmedical casereports.com/content/6/1/427 
Environmental Protection Agency. (2012.) Health Effects. Retrieved from http://www.epa.gov/rpdweb00/understand/health_effects.html

Fanburg-Smith, J. C., Fetsch, J. F., Miettinen, M., Shmookler, B. M., \& Virolainen, M. (1999). Epithelioid Sarcoma: An Immunohistochemical Analysis of 112 Classical and Variant Cases and a Discussion of the Differential Diagnosis. Hum Pathol, 30(8), 934-942.

Hino, O. \& Maeda, M. (2006). Blood Tests for Asbestos-Related Mesothelioma. Oncology, 71, 26-31.

Hino, O. \& Maeda, M. (2006). Molecular Tumor Markers for Asbestos-Related Mesothelioma: Aerum Diagnostic Markers. Pathology International, 56(11), 649-654. doi:10.1111/j.1440-1827.2006.02024.x

Hino, O. \& Shiomi, K. (2007). Diagnostic Biomarker of Asbestos-Related Mesothelioma: Example of Translational Research. Cancer Science 98(8), 1147-1157. doi: 10.1111/j.1349-7006.2007.00520.x

Maniwa, Y., Nishimura, Y., Ohno, Y., Takata, M., \& Yoshimura, M. (2008). "Effective Chemotherapy Based on a Chemosensitivity Test for Malignant Pleural Mesothelioma." Annals Thoracic Surgery, 14, 319-321.

National Cancer Institute. In NCI Dictionary of Cancer Terms. Retrieved from http://www.cancer.gov/dictionary

National Cancer Institute. What is Cancer?. Retrieved from http://www.cancer.gov/ cancertopics/cancerlibrary/what-is-cancer

NDT Resource Center. Radiation: Production of Radiation for Industrial Radiology. Retrieved from https://www.ndeed.org/EducationResources/ CommunityCollege/RadiationSafety/theory/production.htm

Price, A. (2011). "What is the Role of Radiotherapy in Malignant Pleural Mesothelioma?” The Oncologist, 16(3), 359-365. doi:10.1634/theoncologist. 2010-0185

Radiology Masterclass. Basics of X-ray Physics. Retrieved from http://radiology masterclass.co.uk/tutorials/physics/x-ray_physics_production.html

Schittman, G. (2014.) Mesothelioma Symptoms, Causes, Treatment - What Is the Prognosis for Mesothelioma? Retrieved from http://www.medicinenet.com /mesothelioma/page4.htm

Siegel, B. James Rhio O'Connor and His Mesothelioma Story. Retrieved from http://survivingmesothelioma.com/survivors/rhio-o-conor/

Specenier, P. \& Vermorken, J. B. (2011). Cetuximab in the Treatment of Squamous Cell Carcinoma of the Head and Neck. Expert Review of Anticancer Therapy, 11(4), 511-514.

Stabin, M. G. (2013). Doses from Medical Radiation Sources. Retrieved from http://hps.org/hpspublications/articles/dosesfrommedicalradiation.html Surmont, V. F., van Meerbeeck, J. P. \& van Thiel, E. R. (2011). Malignant Pleural 
Mesothelioma: When is Radiation Therapy Indicated? Expert Review of Anticancer Therapy, 11(4), 551-560. doi: 10.1586/era.10.169

The Mesothelioma Center. Resources and Services for Mesothelioma Patients \& Families. Retrieved from http://www.asbestos.com/patient-resources/ United States Department of Labor. Safety and Health Topics: Asbestos. Retrieved from https://www.osha.gov/SLTC/asbestos/ 